\title{
Use of a Plasmodium vivax genetic barcode for genomic surveillance and parasite tracking in Sri Lanka
}

Rajika L. Dewasurendra ${ }^{1}$ (1), Mary Lynn Baniecki², Stephen Schaffner ${ }^{2,3}$, Yamuna Siriwardena', Jade Moon ${ }^{4}$, R. Doshi ${ }^{5}$, Sharmini Gunawardena' ${ }^{1}$, Rachel F. Daniels ${ }^{3}$, Daniel Neafsey ${ }^{2,3}$, Sarah Volkman ${ }^{2,3}$, Naduviladath V. Chandrasekharan ${ }^{6}$, Dyann F. Wirth ${ }^{2,3}$ and Nadira D. Karunaweera ${ }^{1{ }^{*}}$

\begin{abstract}
Background: Sri Lanka was certified as a malaria-free nation in 2016; however, imported malaria cases continue to be reported. Evidence-based information on the genetic structure/diversity of the parasite populations is useful to understand the population history, assess the trends in transmission patterns, as well as to predict threatening phenotypes that may be introduced and spread in parasite populations disrupting elimination programmes. This study used a previously developed Plasmodium vivax single nucleotide polymorphism (SNP) barcode to evaluate the population dynamics of P. vivax parasite isolates from Sri Lanka and to assess the ability of the SNP barcode for tracking the parasites to its origin.

Methods: A total of 51 P. vivax samples collected during 2005-2011, mainly from three provinces of the country, were genotyped for 40 previously identified P. vivax SNPs using a high-resolution melting (HRM), single-nucleotide barcode method. Minor allele frequencies, linkage disequilibrium, pair-wise $F_{S T}$ values, and complexity of infection (COI) were evaluated to determine the genetic diversity. Structure analysis was carried out using STRUCTURE software (Version 2.3.4) and SNP barcode was used to identify the genetic diversity of the local parasite populations collected from different years. Principal component analysis (PCA) was used to determine the clustering according to global geographic regions.

Results: The proportion of multi-clone infections was significantly higher in isolates collected during an infection outbreak in year 2007. The minor allele frequencies of the SNPs changed dramatically from year to year. Significant linkage was observed in sample sub-sets from years 2005 and 2007. The majority of the isolates from 2007 consisted of at least two genetically distinct parasite strains. The overall percentage of multi-clone infections for the entire parasite sample was 39.21\%. Analysis using STRUCTURE software (Version 2.3.4) revealed the high genetic diversity of the sample sub-set from year 2007. In-silico analysis of these data with those available from other global geographical regions using PCA showed distinct clustering of parasite isolates according to geography, demonstrating the usefulness of the barcode in determining an isolate to be indigenous.
\end{abstract}

Conclusions: Plasmodium vivax parasite isolates collected during a disease outbreak in year 2007 were more genetically diverse compared to those collected from other years. In-silico analysis using the 40 SNP barcode is a useful

${ }^{*}$ Correspondence: nadira@parasit.cmb.ac.lk

${ }^{1}$ Department of Parasitology, Faculty of Medicine, University of Colombo,

25, Kynsey Road, Colombo 8, Sri Lanka

Full list of author information is available at the end of the article

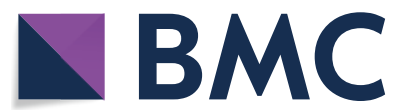

(c) The Author(s) 2020. This article is licensed under a Creative Commons Attribution 4.0 International License, which permits use, sharing, adaptation, distribution and reproduction in any medium or format, as long as you give appropriate credit to the original author(s) and the source, provide a link to the Creative Commons licence, and indicate if changes were made. The images or other third party material in this article are included in the article's Creative Commons licence, unless indicated otherwise in a credit line to the material. If material is not included in the article's Creative Commons licence and your intended use is not permitted by statutory regulation or exceeds the permitted use, you will need to obtain permission directly from the copyright holder. To view a copy of this licence, visit http://creativeco mmons.org/licenses/by/4.0/. The Creative Commons Public Domain Dedication waiver (http://creativecommons.org/publicdomain/ zero/1.0/) applies to the data made available in this article, unless otherwise stated in a credit line to the data. 
tool to track the origin of an isolate of uncertain origin, especially to differentiate indigenous from imported cases. However, an extended barcode with more SNPs may be needed to distinguish highly clonal populations within the country.

Keywords: High-resolution melting method, Genetic diversity, SNP barcode

\section{Background}

Malaria imposed a tremendous health burden in Sri Lanka, until the year 2000 [1, 2] (Fig. 1). The key factors that contributed to the successful reduction of malaria incidence included successful integrated vector control, entomological and parasitological surveillance, thorough epidemiological investigations, disease management, and financial support for diagnostic laboratories and health education [3]. Suppression of control efforts, upon approaching near elimination, often leads to malaria resurgence $[4,5]$, which is clearly evident from past experiences of many countries that include Sri Lanka [1, 2], India [6] and Madagascar [7].

In 2016, the World Health Organization (WHO) certified Sri Lanka as malaria-free. However, as the past predicts, there remains a risk of malaria re-introduction. The last indigenous case of malaria in Sri Lanka was reported in October 2012; however, imported malaria cases continue to be recorded in notable numbers $(95,49,36,41,47$, and 48 cases reported during successive years between 2013 and 2018) [5, 8]. Thus, it is crucial to maintain active surveillance programmes to detect such cases early and contain the situation effectively. Accurate identification of the parasite is critically important in this process in order to make proper patient management decisions especially regarding chemotherapy [9].

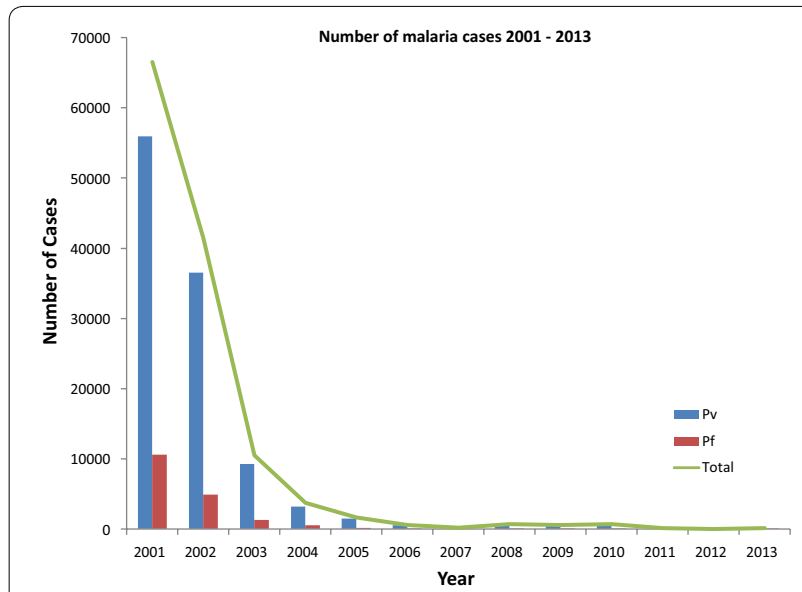

Fig. 1 Number of malaria cases reported 2001-2013. (Source: Anti-malaria Campaign, Ministry of Health, Sri Lanka)
High-resolution melting (HRM) analysis has been used to develop single nucleotide polymorphism (SNP) barcodes for both Plasmodium falciparum and Plasmodium vivax parasites [10, 11]. HRM is a simple and cost-effective method for genotyping and mutation scanning [12, 13]. The malaria molecular barcodes have been shown to have the sensitivity to identify an infection with a single parasite genome or complex (multi-clone infections) parasite infections containing mixtures of parasite genomes within a given sample [9]. Furthermore, genetic barcodes have been used and validated as tools to monitor transmission and to evaluate interventions showing its capacity to make an impact on malaria elimination efforts [14]. The study of the genetic structure/composition of the parasite will be useful in understanding the history of the parasite populations that may enable tracking down the parasites to its origin, as well as providing insights into trends in parasite transmission which are vital and valuable information for elimination programmes [15].

The main objectives of the study described were to investigate the genetic diversity of $P$. vivax parasite isolates collected from patients mainly from three different locations in Sri Lanka over a period of 7 years (20052011), and to test the utility of a SNP barcode in tracking parasites to its origin, which remains particularly important in post-elimination settings, such as in Sri Lanka.

\section{Methods}

\section{Ethical considerations}

Ethics approval for this study was obtained by the Ethics Review Committee, Faculty of Medicine, University of Colombo (Ref No: EC-11-191).

\section{Field isolates}

Before elimination phase, the Anti-malaria Campaign, Ministry of Health, Sri Lanka collected blood samples of all malaria-positive patients through the Regional Malaria Officers from all of the 25 administrative districts in Sri Lanka. According to a mutual agreement between the Anti-malaria campaign and the Department of Parasitology, Faculty of Medicine, University of Colombo, a dried blood spot on filter paper (3MM Whatmann) from these blood samples collected were sent to the laboratory of the Department of Parasitology for research purposes (after obtaining informed consent from the patient), together with the records of the age, gender and location 
of the patient. These patients presented with fever and were diagnosed as having vivax malaria infection confirmed through microscopy in local health facilities. All samples were assumed to be autochthonous at the outset, since a travel history outside the country was not mentioned for any patient.

Fifty-one $P$. vivax field isolates which were sent to the Department of Parasitology, by the Anti-malaria campaign were selected for genotyping (Additional file 1). These isolates had been collected between 2005 and 2011 from the Eastern $(n=22)$, North Western $(n=6)$, Southern $(n=9)$, Uva $(n=1)$, and Northern $(n=1)$ provinces. The origin or location of 12 parasite field isolates was not recorded (Fig. 2).

\section{DNA sample preparation and HRM barcode method}

DNA was extracted from the dried blood spots using a commercial kit (QIAmpBlood Micro Kit-cat\# 56304, QIAGEN, Germany) according to the manufacturer's protocol. Extracted DNA samples (each $1 \mu \mathrm{L}$ ) were subjected to whole genome amplification (WGA) using the IllustraGenomiPhi V2 DNA Amplification kit (GE Healthcare Bio-Sciences, Piscataway, NJ, USA) according to the manufacturer's protocol, yielding $40 \mu \mathrm{L}$ of

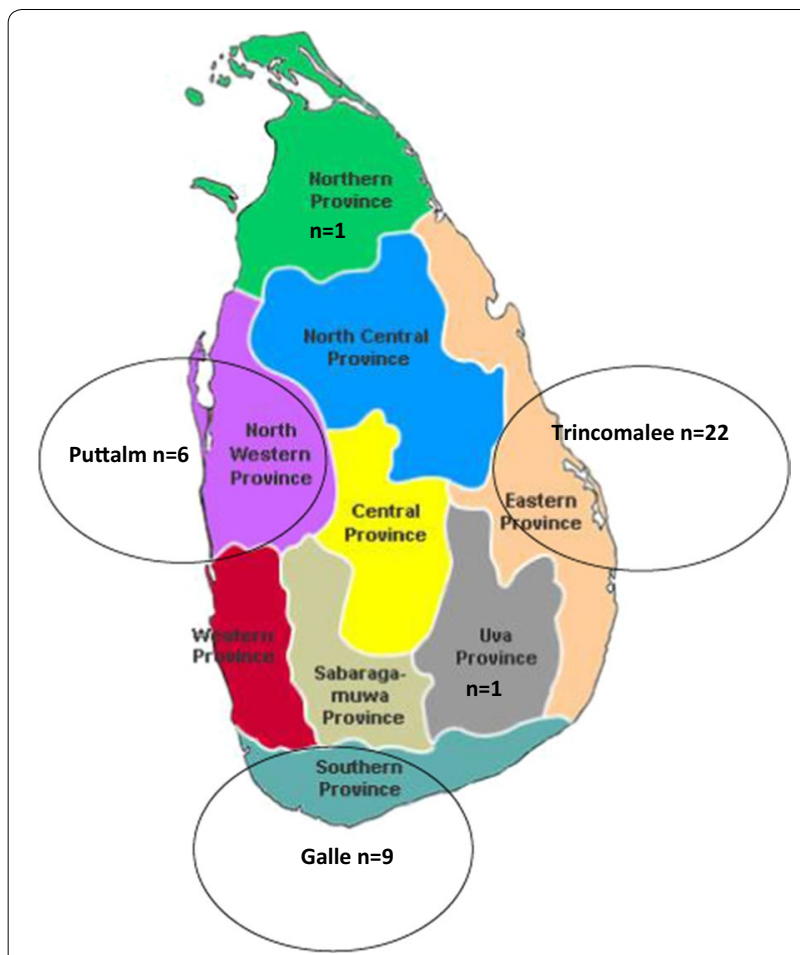

Fig. 2 The locations/districts from where the parasite isolates were collected. The isolates were collected from three districts in Sri Lanka; Trincomalee (Eastern province), Puttalm (North Western province), and Galle (Southern province). The locations of 12 parasite isolates were not recorded amplified material. The amplified DNA was then purified using the AgencourtAMPure SP system (Beckman Coulter, Inc., Beverly, MA, USA) according to the manufacturer's protocol and the samples were quantified using a NanoDrop3300 Fluorospectrometer (Thermo Scientific, Waltham, MA, USA). The samples were then diluted in $1 \times$ Tris-EDTA (TE) Buffer (VWR, Radnor, PA, USA) to a concentration of $1 \mathrm{ng} / \mu \mathrm{L}$.

The $P$. vivax HRM barcode was developed as described by Baniecki et al. [11]. The present study used 40 of the 42 SNPs (Additional file 2). In brief, an HRM master mix was prepared with $1.0 \mu \mathrm{L}$ of forward primer $(1.0-0.5 \mu \mathrm{M} /$ $\mu \mathrm{L}), 1.0 \mu \mathrm{L}$ of reverse primer $(1.0-0.5 \mu \mathrm{M} / \mu \mathrm{L}), 1.0 \mu \mathrm{L}$ of PCR-grade water and $4.0 \mu \mathrm{L}$ of $2.5 \times$ Light Scanner Master mix (BioFire Diagnostics Inc. Salt Lake City, Utah, USA). The optimal primer pair concentration of the forward and reverse primers depended on the individual HRM assays [11]. To each well of a 384-well PCR plate, $7.0 \mu \mathrm{L}$ of the master mix and $3.0 \mu \mathrm{L}$ of template DNA $(1 \mathrm{ng} / \mu \mathrm{L})$ after WGA (details described in Baniecki et al. [11]) were added in a total volume of $10.0 \mu \mathrm{L}$, The plate was sealed with an optically clear, pressure-sensitive adhesive film and was centrifuged at $1000 \mathrm{rpm}$ for $1 \mathrm{~min}$. HRM was performed on a QuantStudio6 Flex Real-Time PCR system (Applied Biosciences). The two-step PCR cycling conditions were as follows:

$95^{\circ} \mathrm{C}$ for $120 \mathrm{~s}, 40$ cycles of $94{ }^{\circ} \mathrm{C}$ for $30 \mathrm{~s}$ and $64{ }^{\circ} \mathrm{C}$ for $60 \mathrm{~s}$ followed by a final HRM cycle of $95^{\circ} \mathrm{C}, 55^{\circ} \mathrm{C}$ and $95^{\circ} \mathrm{C}$ each of $15 \mathrm{~s}$.

\section{Genotype determination}

Method described in Baniecki et al. was used to determine the genotypes [11]. Briefly, a control sample was included for each test sample in the HRM assay to identify the reference and alternate SNP temperature melt-curves $\left(\mathrm{T}_{\mathrm{m}}\right)$ of each SNP in the test samples. The $\mathrm{T}_{\mathrm{m}}$ peak produced by the control sample was aligned with the peaks produced by the test samples to identify each allele. Sample genotypes were identified using the derivative $T_{m}$ curve for each SNP of each sample. The reference/alternate alleles produce a single $T_{m}$ peak that differs $0.7-1.2{ }^{\circ} \mathrm{C}$ and a monomorphic SNP can be identified by aligning this single $T_{m}$ peak with the control $T_{m}$ curve. Since $P$. vivax parasites are haploid in peripheral blood stream identification only a single allele in any SNP position is considered as a monomorphic site, while detection of 2 alleles is considered as a polymorphic site. Polymorphic sites produces skewed or shifted $\mathrm{T}_{\mathrm{m}}$ curves. Samples with more than one polymorphic site in a given SNP position was considered multiclonal/polygenomic [15]. In polymorphic infections, when the isolate consisted of 2 clones, the allele for a given SNP position was determined by comparing the melting curve of the 
isolate with the relevant reference and alternate controls. However, the exact number of clones could not be determined using this method, which enables categorization of an isolate as either single clonal or multi-clone (or polyclonal).

\section{Data analysis}

A total of 51 samples were genotyped by HRM. The samples were divided into 6 sub-sets according to the year of collection, as follows: sub-set of isolates collected in $2005(\mathrm{n}=8), 2006$ sub-set $(\mathrm{n}=1), 2007$ sub-set $(\mathrm{n}=18)$,
2009 sub-set $(\mathrm{n}=4), 2010$ sub-set $(\mathrm{n}=11)$, and 2011 subset $(n=2)$. The year of collection of seven samples were unknown. A genetic barcode for the Sri Lankan vivax malaria parasite was built by arranging SNP data for the 40 SNPs, grouped by each sample sub-set for each year (Fig. 3) and by arranging the SNPs grouped by the location from which the parasite isolates were collected (Additional file 3).

The haplotypes were developed by using Arlequin V 3.1 software by using ELB (Bayesian) algorithm [16] and further confirmed by the COIL web-based tool [17]

\begin{tabular}{|c|c|c|c|c|c|c|c|c|c|c|c|c|c|c|c|c|c|c|c|c|c|c|c|c|c|c|c|c|c|c|c|c|c|c|c|c|c|c|c|}
\hline Province & Year & Clone_Type & Code & $\begin{array}{ll}1 & 2 \\
C & C\end{array}$ & $\begin{array}{l}34 \\
\mathrm{~A} A\end{array}$ & $\begin{array}{ll}5 & 6 \\
\text { C } & A\end{array}$ & $\begin{array}{ll}7 & 8 \\
T & G\end{array}$ & & $\begin{array}{c}10 \\
\mathrm{C}\end{array}$ & $\begin{array}{c}11 \\
\text { C }\end{array}$ & & $\begin{array}{c}13 \\
A\end{array}$ & 14 & C & C & $T$ & G & C & C & T & $\begin{array}{ll}T \\
\text { T }\end{array}$ & C & & & 262 & $T$ & & 29 & & & 32 & G & & & G & & 38 & & $\begin{array}{c}40 \\
C\end{array}$ \\
\hline 99 & 99 & Single & 1 & C C & A G & T G & T G & $T$ & C & C & C & A & C & $T$ & T & $T$ & $T$ & C & $T$ & C & C & $T$ & C & A & A & C & G & $T$ & $T$ & A & $\begin{array}{ll}T \\
\end{array}$ & G & $\mathrm{T}$ & C & G & $\mathbf{T}$ & $\mathbf{T}$ & G & C \\
\hline 99 & 99 & Single & 2 & C C & A A & T G & T G & $T$ & C & C & c & A & C & T & $\mathrm{x}$ & $T$ & $T$ & c & T & c & $x$ & $\mathbf{T}$ & c & A & A & T & G & $T$ & T & A & $x$ & G & C & C & G & $T$ & T & G & C \\
\hline 99 & 99 & Single & 3 & C C & A G & T G & C G & $\mathbf{T}$ & $T$ & C & c & A & C & $\mathrm{T}$ & C & G & G & C & $T$ & C & C & $T$ & c & A & A & T & G & $\mathbf{T}$ & c & G & $T$ & G & C & c & G & $T$ & $T$ & A & C \\
\hline 99 & 99 & Single & 248 & c c & A A & $T G$ & T G & $T$ & C & C & c & A & C & $\mathbf{T}$ & $\mathbf{T}$ & $T$ & $T$ & c & $T$ & c & C & $T$ & c & A & A & $\mathbf{T}$ & G & $\mathbf{T}$ & $T$ & A & $T$ & G & $T$ & C & G & $\mathbf{T}$ & $\mathrm{T}$ & G & C \\
\hline \multirow[t]{2}{*}{99} & 99 & Single & 520 & C C & A A & T G & T G & $T$ & C & C & c & A & C & $T$ & T & $T$ & $T$ & c & $T$ & c & C & $T$ & c & A & A & T & G & T & T & A & $T$ & G & $T$ & c & G & $T$ & T & G & C \\
\hline & 99 & Single & 4793 & C C & A A & $X \mathrm{G}$ & T G & $\mathbf{T}$ & C & C & c & A & C & $\mathbf{T}$ & T & $T$ & $T$ & C & $\mathbf{T}$ & $\mathbf{x}$ & C & $T$ & c & A & A & $\mathbf{T}$ & G & $\mathbf{T}$ & $\mathrm{T}$ & A & $T$ & G & $\mathbf{T}$ & C & G & $\mathrm{x}$ & $\mathrm{T}$ & G & C \\
\hline Southern & 99 & Multi & G15 & C C & A N & X A & C G & $T$ & $T$ & C & c & A & $T$ & $T$ & c & $\mathbf{N}$ & G & $\mathrm{x}$ & $T$ & c & c & c & A & A & A & T & G & T & c & $x$ & $T$ & $\mathbf{N}$ & $T$ & c & G & $T$ & $\mathbf{T}$ & A & $\mathrm{x}$ \\
\hline North Western & 2005 & Single & 5 & C C & A G & T G & C G & $\mathbf{T}$ & T & C & c & A & C & T & C & G & G & $T$ & T & C & T & T & c & A & G & T & G & T & C & G & $T$ & G & C & c & G & $\mathbf{T}$ & T & A & C \\
\hline North Western & 2005 & Single & 6 & c c & A G & T G & C G & & $\mathbf{T}$ & C & c & A & C & $\mathrm{T}$ & C & G & G & T & $T$ & C & $T$ & $T$ & c & A & A & $\mathrm{T}$ & G & $\mathbf{T}$ & C & G & $\mathbf{T}$ & G & C & C & G & 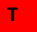 & T & A & C \\
\hline North Western & 2005 & Single & 7 & c c & A G & T G & C G & C & $T$ & C & C & A & C & $T$ & C & G & G & $\mathbf{x}$ & $T$ & C & $T$ & $T$ & c & A & A & $\mathrm{T}$ & G & T & C & G & $T$ & G & C & C & G & $T$ & T & A & C \\
\hline North Western & 2005 & Single & 8 & c c & XA & $T G$ & T G & & C & $\mathbf{T}$ & c & $\mathrm{x}$ & C & T & $T$ & $T$ & $T$ & C & $T$ & c & $x$ & $T$ & c & A & A & $\mathrm{T}$ & $x$ & $T$ & $\mathrm{~T}$ & A & $T$ & G & $T$ & C & G & $T$ & $T$ & G & C \\
\hline North Western & 2005 & Multi & 9 & c c & A A & T G & $\mathrm{T} \mathbf{N}$ & & c & C & c & A & $\mathbf{N}$ & $\mathrm{T}$ & $\mathbf{T}$ & $T$ & $\mathbf{T}$ & c & N & c & C & $T$ & c & A & A & T & G & $\mathrm{T}$ & $T$ & A & $T$ & A & $\mathrm{T}$ & c & G & $T$ & $\mathbf{T}$ & G & $x$ \\
\hline 99 & 2005 & Single & 12 & c c & A A & $T G$ & T G & & c & C & c & A & C & $\mathbf{T}$ & C & $T$ & $\mathrm{~T}$ & c & $T$ & C & C & $T$ & c & A & A & $\mathbf{T}$ & G & $\mathbf{T}$ & $T$ & A & $\mathbf{T}$ & A & $\mathbf{T}$ & C & G & $\mathbf{T}$ & $\mathbf{T}$ & G & C \\
\hline 99 & 2005 & Single & 15 & c c & $A G$ & $T \mathrm{G}$ & T G & & C & C & C & A & C & $T$ & $\mathrm{~T}$ & $T$ & $T$ & C & $T$ & C & C & T & c & A & A & $\mathbf{T}$ & G & $T$ & $T$ & A & T & G & $\mathbf{T}$ & C & G & $\mathbf{T}$ & $\mathrm{T}$ & G & C \\
\hline North Western & 2005 & Multi & 16 & c c & N A & $\mathrm{T} \mathbf{N}$ & $T G$ & & C & c & $\mathbf{N}$ & A & $\mathbf{N}$ & $T$ & $\mathbf{N}$ & $\mathbf{N}$ & $\mathbf{N}$ & c & $\mathbf{N}$ & C & C & $T$ & c & A & A & $\mathbf{T}$ & G & T & T & A & $\mathbf{T}$ & A & $\mathbf{T}$ & C & G & & $\mathrm{x}$ & G & C \\
\hline Eastern & 2006 & Single & 22 & C C & A A & T G & T G & $T$ & C & C & c & A & C & $\mathrm{T}$ & $T$ & $T$ & $T$ & c & $T$ & C & C & $T$ & c & A & A & T & G & $T$ & T & A & $T$ & G & $\mathrm{T}$ & c & G & $T$ & $T$ & G & C \\
\hline 99 & 2007 & Single & 23 & c c & A A & $T G$ & T G & $T$ & C & C & c & A & C & T & $x$ & $T$ & $T$ & C & $T$ & C & C & $T$ & c & A & A & $\mathbf{T}$ & G & $T$ & $\mathrm{~T}$ & A & $x$ & G & $\mathbf{T}$ & C & G & $T$ & $\mathbf{T}$ & G & C \\
\hline 99 & 2007 & Single & 24 & C & A A & $T$ G & T G & $\mathbf{T}$ & C & C & c & A & c & T & T & T & $\mathrm{T}$ & c & $\mathbf{T}$ & c & c & $\mathbf{T}$ & c & A & A & $\mathbf{T}$ & G & $\mathbf{T}$ & $T$ & A & $\mathbf{T}$ & G & $\mathrm{T}$ & c & G & $\mathbf{T}$ & $\mathbf{T}$ & G & c \\
\hline Eastern & 2007 & Single & 25 & c c & A G & T G & T G & $T$ & C & C & C & A & C & $T$ & T & $\mathbf{T}$ & $T$ & C & T & C & C & $T$ & C & A & A & T & G & $T$ & T & A & $\mathrm{T}$ & A & $T$ & C & G & $\mathbf{T}$ & $T$ & G & C \\
\hline Eastern & 2007 & Multi & 34 & $\mathrm{~N} C$ & A G & T A & C G & C & $T$ & C & C & A & c & $x$ & C & G & G & C & $\mathbf{T}$ & C & $x$ & C & A & $N$ & A & $\mathrm{T}$ & G & $T$ & C & G & $T$ & A & C & C & G & $T$ & T & $\mathbf{N}$ & C \\
\hline Eastern & 2007 & Single & G115 & C C & A G & T A & $X \mathrm{G}$ & C & $\mathbf{T}$ & c & $\mathrm{x}$ & A & c & $T$ & C & G & G & $T$ & $T$ & c & C & c & A & A & A & $T$ & G & $\mathrm{T}$ & C & G & $\mathrm{T}$ & G & $T$ & $x$ & G & $T$ & $\mathrm{~T}$ & A & C \\
\hline Eastern & 2007 & Single & $\mathrm{T} 1$ & c c & & $\mathrm{T} A$ & $T G$ & $T$ & C & C & C & A & c & $\mathrm{x}$ & $x$ & $\mathrm{~T}$ & $T$ & $T$ & $T$ & C & C & $T$ & C & A & A & $T$ & G & $\mathrm{T}$ & $T$ & $x$ & $x$ & G & $T$ & C & G & $T$ & $\mathrm{~T}$ & G & C \\
\hline Eastern & 2007 & Multi & S2 pilot screen & N C & A G & T G & N A & & $\mathbf{N}$ & C & $\mathrm{T}$ & A & $\mathrm{T}$ & $\mathrm{T}$ & $\mathbf{N}$ & G & G & $\mathbf{N}$ & $T$ & $T$ & C & $T$ & C & G & G & $\mathrm{T}$ & G & N & $\mathbf{N}$ & A & C & A & $\mathrm{T}$ & C & G & $\mathbf{T}$ & C & G & $\mathbf{N}$ \\
\hline Eastern & 2007 & Multi & S5 pilot screen & C & N G & $\mathrm{T} A$ & $\mathrm{~T} A$ & $\mathrm{~T}$ & C & $\mathbf{N}$ & $\mathbf{N}$ & G & $T$ & $\mathrm{~T}$ & $\mathbf{N}$ & $\mathbf{N}$ & N & C & C & $T$ & c & $\mathbf{N}$ & N & $\mathbf{N}$ & $\mathbf{N}$ & $T$ & G & $\mathbf{N}$ & $\mathbf{N}$ & N & $\mathbf{N}$ & G & $\mathbf{N}$ & C & G & $\mathbf{T}$ & $\mathbf{N}$ & $\mathbf{N}$ & C \\
\hline Eastern & 2007 & Multi & S6 pilot screen & C & G G & N A & C A & 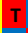 & C & C & C & G & $T$ & T & $T$ & $T$ & G & C & C & $T$ & C & C & C & A & A & $\mathrm{T}$ & G & $T$ & $T$ & G & C & A & $\mathbf{N}$ & C & G & $\mathbf{T}$ & $T$ & G & C \\
\hline Eastern & 2007 & Single & S7 pilot screen & C $\mathrm{T}$ & A G & C G & C A & C & $T$ & C & T & A & C & $T$ & $\mathbf{T}$ & $T$ & G & $T$ & $T$ & $\mathbf{T}$ & $T$ & C & c & A & & c & A & C & C & G & C & G & C & C & G & $T$ & C & G & C \\
\hline Eastern & 2007 & Multi & S8 pilot screen & C T & G G & T G & C A & & $T$ & C & C & A & $T$ & T & C & $T$ & G & C & C & $T$ & C & C & c & A & A & $T$ & G & $\mathbf{N}$ & $T$ & G & C & A & C & C & G & $T$ & $\mathrm{~T}$ & G & C \\
\hline Eastern & 2007 & Multi & S9 pilot screen & $C N$ & $\mathrm{~N} \mathrm{~N}$ & $\mathrm{~N} \mathrm{~N}$ & N A & & $\mathbf{N}$ & $\mathbf{N}$ & $\mathbf{N}$ & $\mathbf{N}$ & $\mathbf{N}$ & $T$ & $\mathbf{N}$ & $T$ & $T$ & C & N & $\mathbf{T}$ & $\mathbf{N}$ & $\mathbf{N}$ & N & A & $\mathbf{N}$ & $\mathrm{T}$ & $\mathbf{N}$ & $\mathbf{N}$ & $T$ & N & C & $\mathbf{N}$ & N & C & G & $T$ & $\mathrm{~T}$ & $\mathbf{N}$ & C \\
\hline Eastern & 2007 & Multi & S10 pilot screen & C C & A A & C A & C A & c & $T$ & $T$ & N & G & C & T & $\mathrm{T}$ & G & G & C & $T$ & $T$ & $T$ & $T$ & c & A & G & C & A & $\mathbf{N}$ & $T$ & A & C & G & $\mathbf{N}$ & C & G & $T$ & $\mathrm{~T}$ & G & C \\
\hline Eastern & 2007 & Multi & S12 pilot screen & $\mathrm{N} \mathrm{C}$ & G G & $T \mathrm{G}$ & C A & $\mathrm{T}$ & $T$ & C & C & $\mathbf{N}$ & $\mathbf{N}$ & C & C & G & G & $T$ & $T$ & $T$ & C & C & c & A & A & T & G & $\mathbf{N}$ & $T$ & G & $T$ & G & $\mathbf{N}$ & $\mathbf{N}$ & G & $\mathbf{N}$ & T & $\mathbf{N}$ & C \\
\hline Eastern & 2007 & Multi & S13 pilot screen & C C & G G & & C A & & C & C & c & A & $T$ & $T$ & C & G & G & $\mathbf{N}$ & $T$ & $T$ & C & C & c & A & A & $\mathrm{T}$ & G & $T$ & $T$ & G & $\mathbf{T}$ & G & C & C & G & $\mathbf{N}$ & C & A & C \\
\hline Eastern & 2007 & Multi & S14 pilot screen & c c & A A & & C G & & $\mathbf{N}$ & C & T & G & C & $T$ & $\mathbf{N}$ & $T$ & G & $T$ & C & c & $\mathbf{N}$ & $T$ & c & A & G & $\mathbf{T}$ & G & $\mathbf{T}$ & $\mathrm{T}$ & G & $\mathrm{T}$ & G & N & C & G & $T$ & $\mathrm{~T}$ & A & c \\
\hline Eastern & 2007 & Single & S16 pilot screen & C C & G A & T G & C G & C. & $T$ & C & $T$ & G & C & $\mathrm{T}$ & $T$ & T & G & $T$ & C & c & $T$ & T & c & A & G & C & G & $T$ & $T$ & G & T & G & C & C & G & $\mathrm{T}$ & $\mathrm{T}$ & A & A \\
\hline Eastern & 2007 & Multi & S17 pilot screen & C C & G G & T A & $T G$ & C & $\mathbf{N}$ & $T$ & C & A & C & $T$ & C & G & G & $T$ & C & C & C & C & c & A & G & C & G & $T$ & C & G & c & A & C & C & G & $T$ & $\mathrm{~T}$ & G & C \\
\hline Southern & 2009 & Single & $\mathrm{G} 23$ & c c & A G & T G & C G & C & $T$ & C & c & A & $T$ & $\mathbf{T}$ & C & G & G & $\mathbf{T}$ & $T$ & C & $T$ & $T$ & c & A & A & $T$ & G & $\mathbf{T}$ & c & G & $T$ & G & $\mathrm{T}$ & C & G & $T$ & $\mathrm{~T}$ & A & c \\
\hline Southern & 2009 & Multi & S1 pilot screen & C & G G & T A & C G & $\mathbf{N}$ & $T$ & C & $T$ & A & $T$ & $\mathbf{T}$ & T & G & G & T & $T$ & C & c & $\mathbf{T}$ & c & A & A & T & G & $\mathrm{T}$ & $\mathbf{T}$ & A & $\mathbf{T}$ & G & $\mathbf{N}$ & C & G & $\mathbf{T}$ & T & G & A \\
\hline Southern & 2009 & Multi & S11 pilot screen & $\mathbf{T}$ & A G & C A & $\mathrm{C} \mathrm{N}$ & C & C & C & C & A & $\mathbf{N}$ & $\mathrm{T}$ & $\mathbf{N}$ & G & G & $\mathbf{N}$ & $T$ & $T$ & C & C & c & A & A & $\mathbf{T}$ & G & $\mathbf{N}$ & $T$ & G & $T$ & G & C & C & G & C & C & A & C \\
\hline Uva & 2009 & Multi & S15 pilot screen & C C & N A & & C G & & $\mathbf{N}$ & C & $T$ & G & C & $\mathrm{T}$ & $\mathbf{N}$ & $T$ & $T$ & $T$ & C & $T$ & $T$ & $T$ & c & A & G & $T$ & G & $T$ & C & G & $\mathrm{T}$ & G & C & c & G & $\mathrm{T}$ & $\mathrm{T}$ & A & A \\
\hline Eastern & 2010 & Single & 90 & C C & A G & $T \mathrm{G}$ & C G & C & $T$ & C & C & A & C & $T$ & C & G & G & $T$ & $\mathrm{~T}$ & C & $T$ & $\mathbf{T}$ & c & A & A & $\mathbf{T}$ & G & $T$ & C & G & $T$ & G & C & A & G & $\mathbf{T}$ & $\mathrm{T}$ & A & C \\
\hline Southern & 2010 & Single & 95 & c c & A A & C A & $T \mathrm{G}$ & $T$ & $T$ & C & c & A & C & $T$ & C & G & G & C & T & C & $T$ & C & c & A & A & $T$ & G & $T$ & $T$ & G & $\mathrm{T}$ & A & c & C & G & C & $\mathbf{T}$ & G & C \\
\hline Southern & 2010 & Multi & MR4775 & C T & G N & $\mathrm{N} \mathrm{N}$ & N A & $c$ & $N$ & $T$ & $T$ & A & $\mathbf{N}$ & C & C & $T$ & $\mathbf{N}$ & C & C & c & $x$ & $N$ & N & $N$ & $N$ & $T$ & $\mathbf{N}$ & $T$ & $T$ & A & $N$ & A & $T$ & $x$ & G & $T$ & T & $N$ & C \\
\hline Eastern & 2010 & Single & $\mathrm{T} 2$ & c c & A A & $T G$ & T G & $T$ & C & C & c & A & C & $T$ & $T$ & $T$ & $T$ & $T$ & $T$ & C & C & $T$ & C & A & A & $T$ & G & $T$ & $T$ & A & $T$ & G & $T$ & C & G & $T$ & $\mathrm{~T}$ & G & C \\
\hline Eastern & 2010 & Single & $\mathrm{T} 4$ & c c & A A & $T G$ & T G & $\mathrm{T}$ & C & C & C & A & $T$ & $T$ & c & $T$ & $T$ & $T$ & T & C & C & T & c & A & A & $\mathbf{T}$ & G & $T$ & $T$ & A & 1 & A & $T$ & c & G & $T$ & $T$ & G & C \\
\hline Eastern & 2010 & Multi & $\mathrm{T7}$ & N C & A N & $T G$ & N G & $\mathrm{N}$ & $\mathbf{N}$ & C & c & A & C & $T$ & $\mathbf{N}$ & $\mathbf{N}$ & $\mathrm{T}$ & C & T & C & C & $\mathbf{T}$ & c & A & A & $\mathbf{T}$ & G & $T$ & $\mathbf{N}$ & $\mathbf{N}$ & $\mathbf{T}$ & G & $\mathbf{N}$ & C & G & $\mathbf{T}$ & $T$ & G & C \\
\hline Southern & 2010 & Single & 620 & c c & A A & $T G$ & $T \mathrm{~T}$ & $T$ & C & C & C & A & C & $\mathrm{T}$ & $\mathrm{T}$ & $T$ & $T$ & C & $T$ & C & C & $\mathbf{T}$ & c & A & A & $\mathbf{T}$ & G & $\mathrm{T}$ & $T$ & A & $\mathrm{T}$ & G & $T$ & C & G & $T$ & $\mathrm{~T}$ & G & C \\
\hline Eastern & 2010 & Single & S3 pilot screen & C & $A G$ & C G & C A & & C & C & $T$ & G & $T$ & $T$ & $\mathbf{T}$ & G & $\mathrm{T}$ & $T$ & C & $T$ & C & C & C & A & G & $\mathbf{T}$ & G & C & $T$ & G & c & A & $\mathrm{T}$ & C & G & $\mathbf{T}$ & $\mathrm{T}$ & A & C \\
\hline Northern & 2010 & Multi & S4 pilot screen & C T & N G & T A & C G & $\mathrm{T}$ & C & C & c & $\mathbf{N}$ & $T$ & $T$ & $T$ & G & G & $T$ & c & $T$ & C & $\mathbf{T}$ & A & G & G & $\mathrm{T}$ & G & $\mathrm{T}$ & C & G & $\mathrm{T}$ & G & N & c & G & $\mathrm{T}$ & T & A & C \\
\hline Southern & 2010 & Single & S18 pilot screen & C C & A G & $\mathrm{TA}$ & $T \mathrm{G}$ & & $T$ & C & $T$ & A & C & $\mathbf{T}$ & C & G & G & C & $\mathbf{T}$ & $\mathbf{T}$ & $T$ & $\mathbf{T}$ & C & G & G & $\mathbf{T}$ & G & C & $\mathbf{T}$ & A & C & A & C & C & G & $\mathbf{T}$ & C & G & C \\
\hline Southern & 2010 & Multi & S19 pilot screen & $\mathbf{N} \mathrm{T}$ & G A & & & & $T$ & $\mathrm{~T}$ & $\mathbf{N}$ & A & C & $T$ & C & $T$ & G & $T$ & $\mathbf{T}$ & $T$ & $T$ & C & c & & & $\mathbf{T}$ & G & $\mathbf{N}$ & T & A & $\mathbf{T}$ & G & C & $\mathbf{N}$ & G & $T$ & c & & A \\
\hline 99 & 2011 & Single & MR4785 & & G G & C A & C A & & C & $T$ & $\mathrm{~T}$ & A & $T$ & C & C & G & G & $x$ & $\mathrm{C}$ & C & C & C & c & & A & 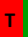 & G & $T$ & $\mathbf{T}$ & A & 1 & A & C & C & G & $\mathrm{T}$ & $\mathbf{T}$ & G & C \\
\hline 99 & 2011 & Single & MR4783 & 0 & & $T G$ & T G & & C & C & C & A & & & & & $\mathrm{T}$ & $c$ & & C & & & & & & & G & $\mathrm{T}$ & & & & G & $\mathrm{T}$ & C & G & $T$ & & G & \\
\hline
\end{tabular}

Fig. 3 Barcodes of parasite isolates grouped together according to year of collection. The location and the year of collection ( $99=$ unknown) for

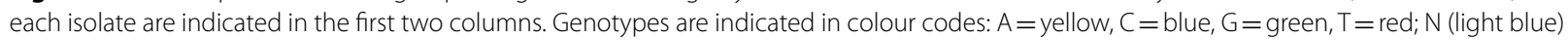
denotes polygenomic sites, and X (light orange) denotes HRM assays that were failed 
which uses a Bayesian model to assign probabilities for SNP barcode data. This program can consider the probability of having a mixture of reference and alternate alleles (multi-clone infections). Furthermore, this program also allows usage of genotypic data with missing information for fewer than 3 loci. The program excludes the samples/isolates with more than 3 loci with missing information. Thus, three samples (one each from the sample sub-sets from 2005 and 2007, and another with unknown year of collection) where excluded from the COIL analysis. Haplotypes were used in generating the $\mathrm{F}_{\mathrm{ST}}$ values to measure the pair-wise distances for determining genetic distance between sample sub-sets.

The minor allele frequency (MAF) was calculated for each polymorphic genotype by counting the reference and alternate alleles, with its average (the unweighted mean) calculated as described by Baniecki et al. [11]. The isolates from multi-clone infections were not considered in calculating the MAF for a particular SNP. These figures for MAF were confirmed by re-calculating the MAF by using the COIL web tool, while retaining the multi-clone isolates designated with an "N call". Although both alleles of multi-clone isolates could be determined by using the differences in the melting curves, the frequency of either major/minor allele could not be determined using the HRM method. Therefore, the positions indicating having 2 or more alleles were designated with an " $\mathrm{N}$ call". These data were retained in analysis by using COIL web-based tool predicting the minimum number of samples present [17]. Loci in linkage disequilibrium (LD) in each group, defined as non-random associations of alleles at each SNP, were calculated for polymorphic SNPs across all 40 SNPs at a significant level of $p<0.05$ with Fisher's exact test using Markov chain (chain length $=10,000$, de-memorization $=10,000$ ) $[18-20]$. Principal component analysis (PCA) was carried out using the online tool ClustVis [21] to compare the genetic structure of parasite isolates from different locations at different time points as well as to compare the genetic structure of parasite isolates collected from Sri Lanka (South Asian, $\mathrm{n}=51$ ) and other geographic regions, i.e., Thailand, Cambodia, Indonesia (Southeast Asian, $\mathrm{n}=181$ ) and Peru (South American, $n=69$ ). The genotypic data were extracted from open access data from MalariaGEN P. vivax genome variation data release 2016 [22] and Cowell et al. 2018 data [23].

All 51 samples were considered for analysis; however, 7 of the 51 samples with unknown years of collection were excluded from linkage analysis and $\mathrm{F}_{\mathrm{ST}}$ estimates. Since there was only one sample from 2006 and two from 2011, they were only included in establishing the barcode since the number of isolates is not a limiting factor for building the barcode. However, they were excluded from other analyses. All 51 samples were used in PCA together with the samples from other global geographic regions.

The genetic distance between the haplotypes was examined by calculating pair-wise genetic distance $\left(\mathrm{F}_{\mathrm{ST}}\right)$. The $\mathrm{F}_{\mathrm{ST}}$ values were obtained using Arlequin V 3.1 software [16] where the genetic distance of pairs of populations were determined using Slatskin's linearized method with 100 permutations with 100,000 steps in Markov chain and 10,000 de-memorization steps [24]. These values were further confirmed by generating $\mathrm{F}_{\mathrm{ST}}$ values using the COIL web-based tool [17]. The significance level was 0.05 . The proportion of multi-clone infections which reflects complexity of infection (COI) was determined to identify the number of genetically distinct parasite strains simultaneously infecting a patient using the COIL program as described previously [17].

A model-based Bayesian method was used to partition of individual parasite isolates into genetic clusters using the STRUCTURE V2.3.4 software [25]. The isolates were grouped into four groups based on the year they were collected (i.e., 2005, 2007, 2009/2010, 2011). The number of clusters (K) was determined by simulating $\mathrm{K}$ values from 1 (with no genetic differentiation among the groups) to 4 (genetic differentiation of each group). The posterior probability values of each $\mathrm{K}$ were estimated from a Markov Chain Monte Carlo (MCMC) method. The multi-clone isolates were excluded in structure analysis.

Additional data analysis was carried out using Excel for Windows 2013, SPSS v 19.0, Genepop v 4.6, and Tassel v 5.2.40 [26-28].

\section{Results}

Genetic barcodes of Sri Lankan Plasmodium vivax parasites The $P$. vivax SNP barcode was used to screen all 51 samples in duplicate. The barcodes were arranged according to the year in which the corresponding isolate was collected (SNP date). The analysis revealed that the isolates collected during the earlier years, i.e., 2005, were less genetically diverse, with more or less constant/shared sub-sets of the haplotypes. In contrast, the sample subset from 2007 looked more diverse and with more multiclone infections (Fig. 3). Secondly, the haplotypes were arranged according to the location from where the corresponding isolates were collected (Additional file 3). It was obvious that the isolates from the Eastern province and the Southern province exhibited a higher genetic diversity with more multi-clone infections compared to the isolates from the North-Western province. Interestingly, the isolates from the Eastern province and the Southern province were collected from short epidemics 
in 2007 from Trincomalee and 2009/10 from Galle districts, respectively.

This genetic barcode was used to distinguish the parasite populations from different geographic regions, i.e., Thailand. Cambodia, Indonesia (Southeast Asian) and Peru (South American) and Sri Lanka (South Asian) using PCA. The isolates from Cambodia, Thailand and Indonesia formed a single cluster, while the Peruvian (South American) and Sri Lankan (South Asian) isolates formed two different clusters (Fig. 4) indicating the possibility of using genetic barcode to track the parasite to its origin. This view was further strengthened through

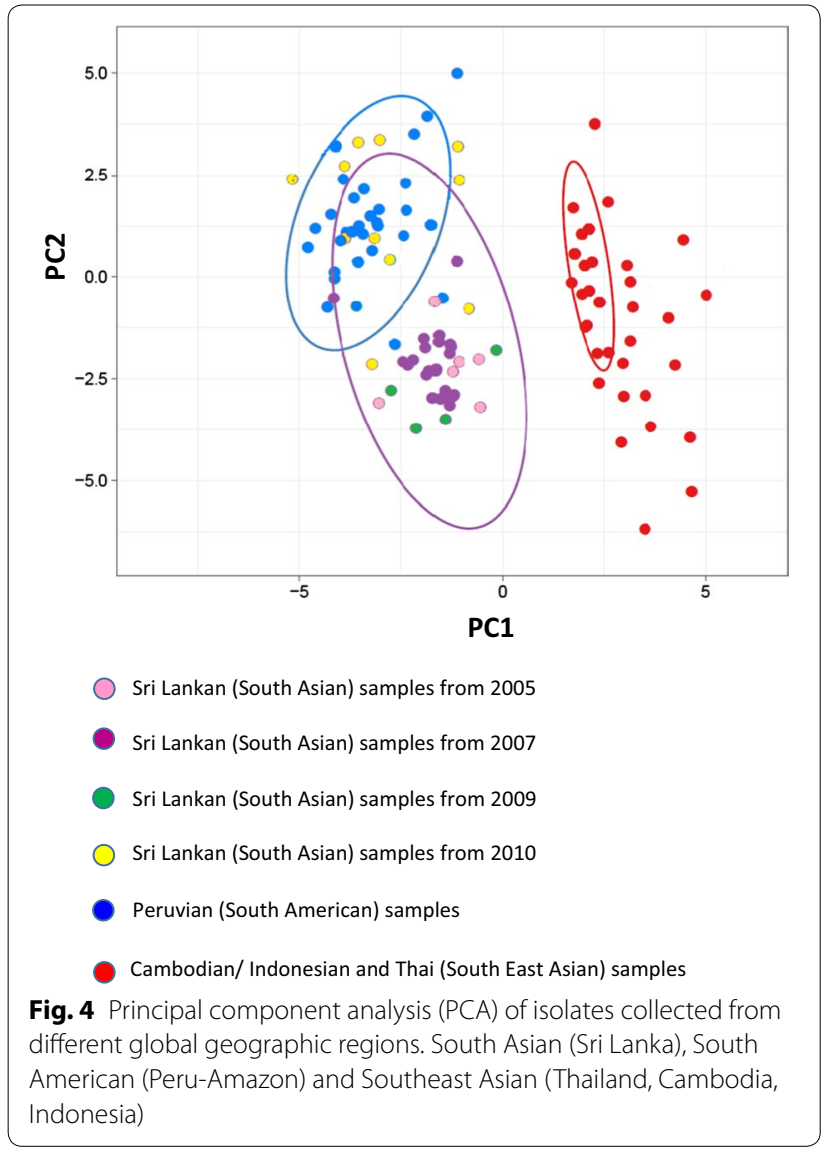

testing of 12 parasite isolates with 'unknown origin' that was found to cluster with the other Sri Lankan samples, confirming that they are likely to be indigenous malaria parasites. Nine of the parasite isolates from Sri Lanka however, clustered with the South American cluster while 2 of the South American isolates were found to cluster with the Sri Lankan group (Fig. 4). Of the 9 isolates that clustered with the South American group, 8 were from the year 2010 (5 from Eastern Province, 2 from Southern Province, 1 from Northern Provinces), and one from year 2007 collected from the Eastern Province outbreak.

However, when only the Sri Lankan isolates were used in PCA, it did not show any clustering according to the year or the location from which the local parasite isolates were collected, potentially due to the limited sample size.

\section{Minor allele frequency (MAF)}

Forty SNPs were studied in each sample sub-set, with the number of polymorphic SNPs varying each year $(10,13$ and 7 polymorphic loci, respectively, in 2005, 2007 and 2010 ; mean $=10.00 \pm 2.49$ ). When the whole population was considered, 32 out of 40 SNPs $(80 \%)$ had minor allele frequencies (MAFs) of $>0.1$. When each sample sub-set was considered, the range of minor allele frequencies of the SNPs differed between the sample sub-sets of different years (from 0.0 to 0.5 ). Basic properties of the genetic nature of the parasite populations are summarized in Table 1.

\section{Complexity of infection (COI)}

Nearly $70 \%$ (69.56\%) of the sample sub-set from 2007 had at least two genetically distinct parasite strains (Table 1), and the proportion of multi-clone infections in the sample sub-set from 2007 was significantly higher $\left(x^{2}=10.227, p=0.017\right.$, adjusted residual \pm 3.1$)$ compared to that in the other years $(25.0 \%$ in 2005 , and $22.22 \%$ in 2010). The overall percentage of multi-clone infections for the entire sample set genotyped was $39.21 \%$. Complexity of Infection (COI) represents the number of genetically distinct parasite strains simultaneously infecting a patient. The mean COI was 1.142, 1.25 and 1.136 for each sample sub-set from 2005, 2007 and 2010,

Table 1 Genetic characteristics of parasite isolates collected between 2005-2011

\begin{tabular}{llll}
\hline & $\mathbf{2 0 0 5}$ & $\mathbf{2 0 0 7}$ & $\mathbf{2 0 1 0}$ \\
\hline No of isolates & 8 & 23 & 40 \\
No of SNPs & 40 & 40 & $69.56 \%$ \\
Proportion of multi-clone infections & $25 \%$ & $4.771( \pm 2.416)$ \\
$\begin{array}{l}\text { Mean number of pair-wise differences (in nucleotides } \\
\quad \pm \text { SD) }\end{array}$ & $4.821( \pm 2.631)$ & $1.400( \pm 0.934)$ \\
\begin{tabular}{l} 
Mean COI \\
\hline
\end{tabular}
\end{tabular}


respectively. Furthermore, out of 40 loci 37 (92.5\%) were polymorphic when polyclonal samples were considered. This was comparable with the percentage of polymorphic loci in monoclonal samples (95\%).

\section{Linkage disequilibrium (LD)}

Significantly high $(\mathrm{p}<0.05)$ LD was observed between several polymorphic loci of the sample sub-sets collected from 2005 and 2007. The maximum numbers of linked loci per polymorphic loci were 6 and 3 in these years, respectively. None of the polymorphic loci was in linkage to the sample sub-set from 2010.

\section{Genetic distances of the sample sub-sets from different years}

The pair-wise $\mathrm{F}_{\mathrm{ST}}$ values estimated for all SNPs indicated that the sample sub-set from 2007 that originated from an outbreak in Trincomalee district differed significantly $(\mathrm{p}<0.05)$ from the sample sub-sets from 2005 and 2010 (Fig. 5).

\section{Genetic structure of sample sub-sets}

The structure analysis, which indicates the proportion of ancestry in each isolate, also revealed that sample sub-sets from the short epidemics 2007 (cluster 2) and 2009/10 (clusters 3 and 4) were more genetically different and diverse when compared to the sample sub-set from the early years, i.e., 2005 (Fig. 6).

\section{Discussion}

The parasite isolates for this study were collected from 2005-2011 mainly from three provinces in Sri Lanka during the period of declining vivax malaria transmission. The genetic composition of a parasite population over time is known to reflect the changes in transmission

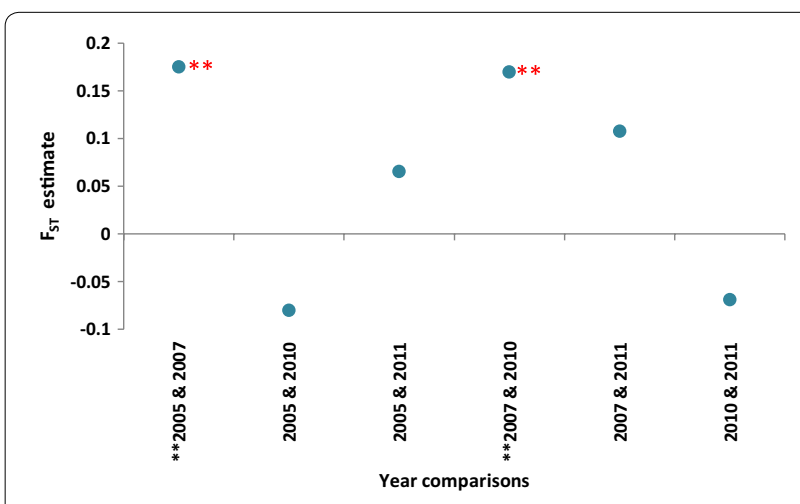

Fig. 5 Pair-wise $F_{S T}$ values obtained for the parasite sub-populations for each year. The parasite populations from 2007 showed a significant difference in their genetic make-up when compared with the populations from years 2005 and 2010

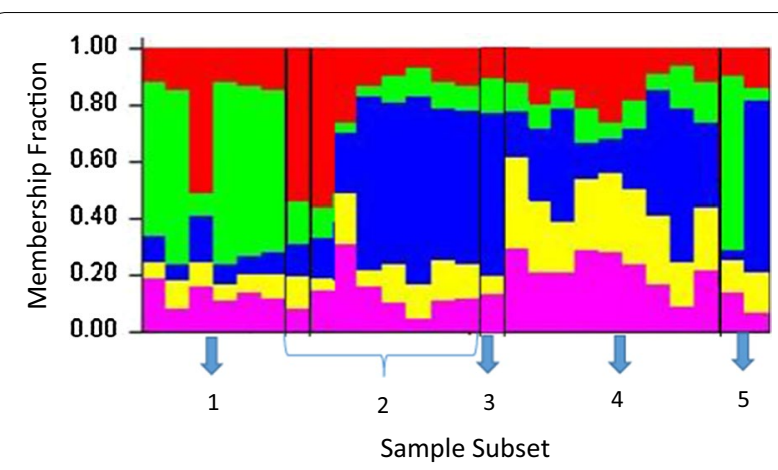

Fig. 6 The population structure (the membership fraction for each sample sub-set) of Plasmodium vivax plotted according to the year in which the isolates were collected inferred from genotyping of parasite isolates (at $K=5$ ). Each bar represents an isolate. Cluster 1-isolates from $2005(n=6)$, cluster 2-isolates from $2006(n=1)$ and $2007(n=7)$, cluster 3-isolates from $2009(n=1)$, cluster 4-isolates from $2010(n=8)$ and cluster 5-isolates from $2011(n=2)$. Seven isolates for which the year of collection is unknown and isolates with multi-clone infections $(n=19)$ were excluded from structure analysis

intensity especially in P. falciparum. However, the genetic information derived of parasite populations could be of limited use in assessing population dynamics and transmission intensity in high transmission areas due to frequent multi-clonal infections (super infections) compared to low transmission areas [29]. On the other hand, the quality of the genetic data depends on the method of genotyping, i.e., although SNP barcoding method could be affected by the presence of high multi-clonal parasite populations, some methods, such as amplicon deep sequencing are not affected [30].

In the present study, the local $P$. vivax parasite population showed an overall high level of genetic diversity based on the 40-SNP barcode, COI and structure analysis, which was viewed in the context of low malaria transmission that prevailed during the study period. This confirms the observations made previously in Sri Lankan $P$. vivax populations using microsatellite markers [31]. Similarly in other geographical regions, it has been shown (using SNP markers) that $P$. vivax tends to maintain a higher genetic diversity even in areas with low transmission. Cui et al. suggested that observation could be due to a number of factors, including intrinsic biological properties of the vivax malaria parasite, such as relapses and early gametocytaemia which favour the cross-fertilization and meiotic recombination of distinct parasite genotypes within the vector. Furthermore, though rare, this genetic diversity could also be influenced by imported parasite strains carried by infected immigrants, as this can increase the genetic variability of the parasites through recombination in the mosquitoes [32]. The parasite isolates in the present study were 
collected during a period in which intense control programmes, especially residual spraying of insecticides for vector control, were in place throughout endemic areas of Sri Lanka. Consequently, the behaviour and distribution of vector mosquitoes may have been affected, which may have influenced the length of the extrinsic parasite life cycle, ultimately influencing the genetic diversity of the parasite population [33]. However, the connection between genetic diversity and the transmission intensity of malaria could be complex, with these two components having a non-linear relationship [34].

High LD is always prominent in areas with lower transmission intensities where genetic diversity is low, as the linkage of markers across chromosomes usually happens when the rate of sexual recombination is low. Sample sub-sets from 2005 and 2007 in this study demonstrated such a pattern. The co-occurrence of high LD with multiclones under decreased transmission intensity was however unusual, although described previously as well by Karunaweera et al. and Gunawardena et al. using 14 polymorphic microsatellite markers [31,35]. The results of the present study further confirm these previous observations. This scenario has also been observed in parasite populations in Brazil [36]. Therefore genetic linkage, especially in low transmission settings, can be a valuable tool to track parasites in populations both temporally and spatially [29].

One of the main findings in this study is that the local parasite population had a high proportion of multiclone infections. Multi-clone infections arise when an individual is simultaneously infected by more than one parasite genotype. This can be due to co-infection of different parasite strains, viz. super infection, which normally occurs with high endemicity or as speculated by Cui et al. through somatic mutations during the course of infection [32]. Nearly $70 \%$ of parasite isolates in the sample sub-set from 2007 were multiclone infections despite the reduced malaria endemicity. However, when the entire $P$. vivax parasite sample set was considered $(n=51)$, the overall percentage of multi-clone infections was $39.21 \%$. This is a relatively lower value compared to those in other geographical areas with relatively higher vivax malaria transmission rates than in Sri Lanka, that include Vietnam (71.4\%) [37], Colombia (60-80\%) [38], and the Amazon Basin of Brazil (50\%) [39]. However, the percentage multiclone infections obtained for sample sub-sets from individual years (apart from the sample sub-set from 2007) and for the total sample set used were comparable to those observed in places with similar transmission dynamics ( $P$. vivax), i.e., in low endemicity areas in South Asia, with $25.8 \%$ in some parts of Malaysia [40]; in post-intervention sites including Solomon
Islands (27\%) [41], Peruvian Amazon (34.7\%) [42], and also in low-endemicity areas in South America with P. falciparum infections [43, 44]. In contrast, previous studies in Sri Lanka using microsatellite markers by Karunaweera et al. in 2007/2008 and Gunawardena et al. in 2014 reported higher percentages $(60,52$, and $68 \%$, respectively) of multi-clone infections [32, 34, 45]. Those samples however had been collected during 2003-2007, when malaria transmission was still relatively higher than the period during which the samples were collected for this study. The samples included from year 2010 had 22\% multi-clone infections, during which time the transmission intensity was very low. These lower values may have contributed to the overall lower percentage of multi-clone infections in this parasite population. Moreover, relapses due to hypnozoites also could account for multi-clone infections in vivax malaria. However, the relapse rates of vivax malaria in these study sites are not well studied to infer the impact of relapses on multi-clone infections. Furthermore, a 2-week course of primaquine was widely used as a radical cure for vivax malaria in Sri Lanka, especially during the malaria pre-elimination phase; eliminating hypnozoites and thus preventing relapses, may have accounted for the low number of multi-clone infections during later years.

The use of several molecular markers increases the chance of detection of multi-clone infections compared to using a few or a single marker $[31,46]$.

The 40-SNP barcode (Fig. 3 and Additional file 3) revealed the presence of common alleles (haplotypes/ barcode types) in parasite clusters from different years and in clusters from different areas, indicating a conserved and/or global distribution of certain parasite genotypes. Each isolate studied possessed a unique haplotype, but shared/common sub-sets of the barcode could be observed between the isolates within the same year as well as between the years. Having the shared/ common sub-set of the barcodes in many isolates could be due to either clonal propagation or epidemic expansion (or both). Although the barcode SNPs on their own are not subjected to selection, the parasite lineages might be associated with other phenotypes that may be under selection pressure, e.g., reproductive success (gametocyte formation, multiplying or recombining inside the vector, etc.). Furthermore, the immune factors in humans (e.g., lack of immunity against a genotype that causes rapid expansion without any association with anti-malarial usage) or other stochastic events, could also affect the allele frequencies of those SNPs [14]. The geographical and temporal distributions of selected parasite genotypes might also be influenced by the seasonal/temporal population dynamics of host anopheline mosquito strains, as 
reported by previous studies describing differences in the susceptibilities of vector mosquitoes to different genetic strains of the parasite [47].

Patient details available did not specify any travel outside of the country for any patient from whom these samples were obtained and, therefore, the isolates were assumed to be indigenous. Out of the 39 isolates with records of the location from which they were collected, 30 isolates were included in a single cluster in PCA, suggesting having a similar genetic structure. There were 12 isolates of which the location of the patient was unknown and in the PCA these isolates clustered with the other Sri Lankan samples, supporting the assumption that those also could be indigenous isolates. Interestingly, 9 isolates (out of which 8 were from year 2010) clustered together with the South American group. It could be speculated that these isolates were imported cases. However, they have been reported from 3 provinces (although the majority were from the Southern province, $n=5$ ). Since no travel histories were indeed available, such speculations remain unconfirmed.

Although the 40-SNP barcode revealed clustering of genetically similar parasites when compared with isolates from other global geographic regions by PCA, it could not distinguish prominent parasite clusters geographically or temporally within the Sri Lankan isolates, indicating the genetic similarities of the parasite isolates used in the analysis. Interestingly, the sample sub-set from 2007, which was collected during a short-term malaria outbreak in Trincomalee, was more genetically diverse compared to the parasite populations from other years. Sri Lanka is a small island, with relatively low physical and socio-demographic barriers, which enables mixing of parasites with different genetic profiles; thus, there is limited potential to preserve geographically isolated parasite populations with distinguishable genetic features. However, this may account for the high genetic diversity in Sri Lankan $P$. vivax populations. Therefore, the 40-SNP barcode may not be sensitive enough to capture the finer scale diversity among highly clonal parasite isolates, as reported in this study. Further studies warrant the development of an extended barcode comprising a greater number of SNPs to differentiate regional parasites with high rate of multi-clone infections.

The origin of a focal outbreak is likely to be a common source and therefore, one might expect to find genetically similar parasites in such a setting. However, the study findings indicated a higher level of genetically diverse groups of isolates within the outbreak when compared to the sample sub-sets from other years. The decreased diversity of sample sub-sets from 2005, 2010, and 2011 may have been influenced by the low transmission/endemicity, with fewer opportunities for sexual recombination between genetically distinct parasites circulating during that period, while the parasite population from the short epidemic (in 2007) may have had a higher chance of recombination with genetically diverse parasites during the short epidemic that occurred in the Trincomalee district, resulting in the high level of genetic diversity observed in this sample sub-set from 2007. Gunawardena et al. in 2010 used microsatellite markers to study the genetic diversity of the vivax isolates from Sri Lanka, reporting that isolates collected during the 2007 malaria outbreak in Trincomalee district demonstrated distinct differences in their genetic make-up compared to that in parasite isolates collected during other years [31]. The results of the current study confirm this observation.

One of the major drawback of this study is the limited numbers contained within the 2006 and 2011 sub-sets, i.e., one sample and two samples, respectively, in 2006 and 2011. However, those sample sub-sets were largely excluded from the analyses, but used only in selected analyses (viz. construction of the genetic barcode) where the outcome is independent of the sample number contained within the sub-set. Furthermore, the information derived from haplotype analyses and $\mathrm{F}_{\mathrm{ST}}$ estimates in this study might not be very informative owing to the small sample size and the high variation of allele frequencies observed between the sample sub-sets from different years. Therefore, future studies with a large number of samples will warrant more accurate and robust results.

More importantly, this study demonstrates the potential significance of using genetic barcodes to aid in distinguishing parasite populations, enabling tracking of imported malaria cases that pose a threat during the post-malaria elimination phase in Sri Lanka and elsewhere.

\section{Conclusions}

This study provides supportive evidence for the potential utility of a genetic barcode that could aid in confirming indigenous malaria cases. In-silico analysis revealed that isolates with unknown origin clustered with Sri Lankan isolates in PCA, supporting the likelihood of these samples being indigenous. This also provides the ability to identify imported malaria cases that pose a threat during the post-malaria elimination (prevention of reintroduction) phase in Sri Lanka. The high percentages of multi-clone infections in the sample sub-sets and the mean COI values/analysis with STRUCTURE (V 2.3.4) software suggests an overall high level of genetic diversity within an environment of low malaria transmission that prevailed during the study period. This effect was more pronounced during the short malaria epidemic in 2007. However, studying the parasite variations within the country needs a more sensitive barcode, probably 
with a higher number of SNPs to distinguish between the indigenous populations. However, the 40-SNP P. vivax barcode was able to identify common/shared sub-sets of barcodes in parasite sample sub-sets from different years and within sample sub-sets from different areas.

\section{Supplementary information}

Supplementary information accompanies this paper at https://doi. org/10.1186/s12936-020-03386-3.

Additional file 1. Details of the parasite isolates used in the study; ID number, year and location of collection, and clonality.

Additional file 2. Details of the genotyped SNPs; SNP ID, chromosome position, gene ID and the genetic properties/effect.

Additional file 3. SNP genetic barcode; Genotyped SNPs arrange according to the place of collection.

\section{Abbreviations}

WHO: World Health Organization; HRM: High-resolution melting; SNP: Single nucleotide polymorphism; PCR: Polymerase chain reaction; WGA: Whole genome amplification; $T_{m}$ : Melting temperature; MAF: Minor allele frequency;

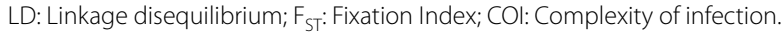

\section{Acknowledgements}

We thank Dr. R. Abeysinghe and G. Galappaththi, Anti-malaria Campaign of the Ministry of Health in Sri Lanka for providing the parasite isolates, Kian Sani and Cynthia of The Broad Institute, Boston MA, USA for help with performing the laboratory work. This publication uses data from the MalariaGEN P. vivax Genome Variation project, as described in Pearson et al., Nature Genetics, 2016 (https://doi.org/10.1038/ng.3599) and P. vivax genome Illumina sequencing reads (Cowell et al., Genome Medicine, 2018 (https://doi.org/10.1186/s1307 3-018-0563-0) available through National Center for Biotechnology Information's Short Read Archive (Accession number SRP095853). Dr. Richard Pearson, Sanger Institute, UK and Dr. Annie Cowell, University of California, San Diego, USA are greatly appreciated for sharing genotypic data of these P. vivax samples. The authors would like to add a note of appreciation for the reviewers who provided critical comments and suggestions for the earlier drafts, which helped to improve this manuscript.

\section{Authors' contributions}

RLD: Funding acquisition, performed lab work, data analysis, and wrote the first draft of the manuscript; MLB: performed lab work, validated the P. vivax barcode, and drafted and provided critical review of the manuscript; SS: performed data analysis and critical review of the manuscript; HVYDS: performed lab work and manuscript review; JM, RD: validated the barcode assay; GSAG: drafted and provided critical review of the manuscript; RFD: performed data analysis and provided critical review of the manuscript; DN: performed data analysis; SV, NVC, DFW: provided critical review of the manuscript; NDK: conceived and provided overall supervision of the project, funding acquisition, and drafting and critical review of the manuscript. All authors read and approved the final manuscript.

\section{Funding}

Funding for this research was provided by World Class University Grants (AP/3/2011/PG/15) and The Broad Institute, Cambridge, MA, USA.

\section{Availability of data and materials}

The datasets used and/or analysed during the current study are available from the corresponding author on reasonable request.

\section{Ethics approval and consent to participate}

Ethics approval for this study was granted by the Ethics Review Committee, Faculty of Medicine, University of Colombo (EC-11-191).

\section{Consent for publication}

Not relevant.

\section{Competing interests}

The authors declare that they have no competing interests.

\section{Author details}

${ }^{1}$ Department of Parasitology, Faculty of Medicine, University of Colombo, 25, Kynsey Road, Colombo 8, Sri Lanka. ${ }^{2}$ Infectious Disease and Microbiome Program, Broad Institute of MIT and Harvard, Cambridge, MA 02142, USA. ${ }^{3}$ Department of Immunology and Infectious Diseases, Harvard T. H. Chan School of Public Health, Boston, MA 02115, USA. ${ }^{4}$ Department of Organismic and Evolutionary Biology, Harvard University, Cambridge, Boston, MA 02138, USA. ${ }^{5}$ Department of Public Health, John Hopkins University, Baltimore, MD 21218, USA. ${ }^{6}$ Department of Chemistry, Faculty of Science, University of Colombo, Munidasa Kumaratunga MW, Colombo 3, Sri Lanka.

Received: 1 May 2019 Accepted: 25 August 2020

Published online: 21 September 2020

\section{References}

1. Karunaweera ND, Galappaththy GN, Wirth DF. On the road to eliminate malaria in Sri Lanka: lessons from history, challenges, gaps in knowledge and research needs. Malar J. 2014;13:59.

2. Abeysinghe RR, Galappaththy GNL, Kahn JG, Feachem RGA, Smith GC. Malaria control and elimination in Sri Lanka: documenting progress and success factors in a conflict setting. PLoS ONE. 2012;7:e43162.

3. WHO, University of California San Francisco. Eliminating malaria: casestudy 3-progress towards elimination in Sri Lanka. Geneva: World Health Organization; 2012.

4. Cohen J, Smith DL, Cotter C, Ward A, Yamey G, Sabot O, et al. Malaria resurgence: a systematic review and assessment of its causes. Malar J. 2012;11:122.

5. Global Health Group. The risk of malaria resurgence. 2014;(September):1-4. https://globalhealthsciences.ucsf.edu/sites/default/files/conte nt/ghg/mei-risk-of-malaria-resurgence.pdf.

6. Sharma VP. Re-emergence of malaria in India. Indian J Med Res. 1996;103:26-45.

7. Randrianarivelojosia M, Raveloson A, Randrianmanantena A, Juliano JJ, Andrianjafy $T$, Raharimalala LA, et al. Lessons learnt from the six decades of chloroquine use (1945-2005) to control malaria in Madagascar. Trans R Soc Trop Med Hyg. 2009;103:3-10.

8. Anti-malaria Campaign Sri Lanka. Anti malaria campaign, Ministry of Health, Sri Lanka. https://www.malariacampaign.gov.lk/Precentation/ AnnualReports.aspx.

9. Daniels R, Volkman SK, Milner DA, Mahesh N, Neafsey DDE, Park DJ, et al. A general SNP-based molecular barcode for Plasmodium falciparum identification and tracking. Malar J. 2008;7:223.

10. Preston MD, Campino S, Assefa SA, Echeverry DF, Ocholla H, AmambuaNgwa A, et al. A barcode of organellar genome polymorphisms identifies the geographic origin of Plasmodium falciparum strains. Nat Commun. 2014;5:4052.

11. Baniecki ML, Faust AL, Schaffner SF, Park DJ, Galinsky K, Daniels RF, et al. Development of a single nucleotide polymorphism barcode to genotype Plasmodium vivax infections. PLoS Negl Trop Dis. 2015;9:e0003539.

12. Gundry CN, Vandersteen JG, Reed GH, Pryor RJ, Chen J, Wittwer CT. Amplicon melting analysis with labeled primers: a close tube method for differentiating homozygotes and heterozygotes. Clin Chem. 2003:49:396-406

13. Wittwer CT, Reed GH, Gundry CN, Vandersteen JG, Pryor RJ. High-resolution genotyping by amplicon melting analysis using LCGreen. Clin Chem. 2003:49:853-60.

14. Daniels R, Chang H, Sene PD, Park DC, Neafsey DE, Schaffner SF, et al. Genetic surveillance detects both clonal and epidemic transmission of malaria following enhanced intervention in Senegal. PLOS ONE. 2013;8:e60780.

15. Carter TE, Malloy H, Existe A, Memnon G, St. Victor Y, Okech BA, et al. Genetic diversity of Plasmodium falciparum in Haiti: insights from microsatellite markers. PLoS ONE. 2015;10:e0140416. https://doi.org/10.1371/ journal.pone.0140416. 
16. Zilversmit M, Harti DL. Evolutionary history and population genetics of human malaria parasite. In: Sherman I, editor. Molecular approaches to malaria. ASM Press: Washington DC; 2005. p. 95-109.

17. Galinsky K, Valim C, Salmier A, De Thoisy B, Musset L, Legrand E, et al. COIL: a methodology for evaluating malarial complexity of infection using likelihood from single nucleotide polymorphism data. Malar J. 2015;14:4.

18. Slatkin M. Linkage disequilibrium-understanding the evolutionary past and mapping the medical future. Nat Rev Genet. 2008;9:477-85.

19. Slatkin $M$, Excoffier $L$. Testing for linkage disequilibrium in genotypic data using the expectation- maximization algorithm. Heredity. 1995;76:377-83.

20. Lewontin R, Kojima K. The evolutionary dynamics of complex polymorphisms. Evolution. 1960;14:458-72.

21. Metsalu T, Vilo J. ClustVis: a web tool for visualizing clustering of multivariate data using principal componant analysis and heatmap. Nucleic Acids Res. 2015;43:W566-W570570.

22. Pearson R, Amato R, Auburn S, Miotto O, Almagro-Garcia J, Amaratunga $C$, et al. Genomic analysis of local variation and recent evolution in Plasmodium vivax. Nat Genet. 2016:48:959-64.

23. Cowell AN, Valdivia HO, Bishop DK, Winzeler EA. Exploration of Plasmodium vivax transmission dynamics and recurrent infections in the Peruvian Amazon using whole genome sequencing. Genome Med. 2018;10:52.

24. Excoffier LG, Schneider S. Arlequin ver. 3.0: an integrated software package for population genetics data analysis. Evol Bioinform Online. 2005:1:47-50.

25. Slatkin M, Voelm L. Fst in a hierarchical island model. Genetics. 1991:127:627-9.

26. IBM Corp. Released 2010. IBM SPSS statistics for Windows, Version 19.0. Armonk: IBM Corp.

27. Raymond M, Rousset F. GenePop (v4.2): population genetics software for exact tests and ecumenicism. J Hered. 1995;86:248-9.

28. Bradbury PJ, Zhang Z, Kroon DE, Casstevens TM, Ramdoss Y, Buckler ES. TASSEL: software for association mapping of complex traits in diverse samples. Bioinformatics. 2007;23:2633-5.

29. Branch OH, Sutton PL, Barnes C, Castro JC, Hussin J, Awadalla P, et al. Plasmodium falciparum genetic diversity maintained and amplified over 5 years of a low transmission endemic in the peruvian amazon. Mol Biol Evol. 2011;28:1973-86.

30. Zhong D, Koepfli C, Cui L, Yan G. Molecular approaches to determine the multiplicity of Plasmodium infections. Malar J. 2018;17:172.

31. Gunawardena S, Karunaweera ND, Ferreira MU, Phone-Kyaw M, Pollack RJ, Alifrangis $M$, et al. Geographic structure of Plasmodium vivax: microsatellite analysis of parasite populations from Sri Lanka, Myanmar, and Ethiopia. Am J Trop Med Hyg. 2010;82:235-42.

32. Cui L, Mascorro CN, Fan Q, Rzomp KA, Khuntirat B, Zhou G, et al. Genetic diversity and multiple infections of Plasmodium vivax malaria in western Thailand. Am J Trop Med Hyg. 2003;68:613-9.

33. Karunaweera N, Ferreira M, Munasinghe A, Barnwell J, Collins W, King $C$, et al. Extensive microsatellite diversity in the human malaria parasite Plasmodium vivax. Gene. 2008;410:105-12.
34. Paul R, Hackford I, Brockman A, Muller-Graf C, Price R, Luxemburger C, et al. Transmission intensity and Plasmodium falciparum diversity on the northwestern border of Thailand. Am J Trop Med Hyg. 1998;58:195-203.

35. Karunaweera N, Ferreira M, Hartle D, Wirth D. Fourteen polymorphic microsatellite DNA markers for the human malaria parasite Plasmodium vivax. Mol Ecol Notes. 2007;7:172-5.

36. Ferreira MU, Karunaweera ND, da Silva-Nunes M, da Silva NS, Wirth DF, Hartl DL. Population structure and transmission dynamics of Plasmodium vivax in rural Amazonia. J Infect Dis. 2007;195:1218-26.

37. Van HN, Delgado-Ratto C, Thanh PV, Van den Eede P, Guetens P, Binh NTH, et al. Population genetics of Plasmodium vivax in four rural communities in central Vietnam. PLoS Negl Trop Dis. 2016;10:e0004434.

38. Imwong M, Nair S, Pukrittayakamee S, Sudimack D, Williams JT, Mayxay M, et al. Contrasting genetic structure in Plasmodium vivax populations from Asia and South America. Int J Parasitol. 2007;37:1013-22.

39. Batista C, Barbosa S, Bastos MDS, Viana S, Ferreira M. Genetic diversity of Plasmodium vivax over time and space: a community-based study in rural Amazonia. Parasitology. 2015;142:374-84.

40. Abdullah NR, Barber BE, William T, Norahmad NA, Satsu UR, Muniandy PK, et al. Plasmodium vivax population structure and transmission dynamics in Sabah Malaysia. PLoS ONE. 2013;8:e82553.

41. Quah YW, Waltmann A, Karl S, White MT, Vahi V, Darcy A, et al. Molecular epidemiology of residual Plasmodium vivax transmission in a paediatric cohort in Solomon Islands. Malar J. 2019;18:106.

42. Delgado-Ratto C, Gamboa D, Soto-Calle VE, Van den Eede P, Torres E, Sánchez-Martínez $L$, et al. Population genetics of Plasmodium vivax in the Peruvian Amazon. PLoS Negl Trop Dis. 2016;10:e0004376.

43. Machado RLD, Voa MMP, Calvosa VSP, Ferreira MU, Rossit ARB, dos Santo EJM, et al. Genetic structure of Plasmodium falciparum populations in Brazilian Amazon region. J Inf Dis. 2004;190:1547-55.

44. Anderson TJC, Haubold B, Williams JT, Estrada-Franco JG, Richardson L, Mollinedo R, et al. Microsatellite markers reveal a spectrum of population structure in the malaria parasite Plasmodium falciparum. Mol Biol Evol. 2000;17:1467-82

45. Gunawardena S, Ferreira MU, Kapilananda GMG, Wirth DF, Karunaweera ND. The Sri Lankan paradox: high genetic diversity in Plasmodium vivax populations despite decreasing levels of malaria transmission. Parasitology. 2014;141:880-90.

46. Li YC, Wang GZ, Meng F, Zeng W, He CH, Hu XM, et al. Genetic diversity of Plasmodium vivax population before elimination of malaria in Hainan Province, China. Malar J. 2015;14:78.

47. Gonzalez-Ceron L, Rodriguez MH, Nettel JC, Villarreal C, Kain KC, Hernandez JE. Differential susceptibilities of Anopheles albimanus and Anopheles pseudopunctipennis to infections with coindigenous Plasmodium vivax variants VK210 and VK247 in Southern Mexico. Infect Immun. 1999;67:410-2.

\section{Publisher's Note}

Springer Nature remains neutral with regard to jurisdictional claims in published maps and institutional affiliations.

Ready to submit your research? Choose BMC and benefit from

- fast, convenient online submission

- thorough peer review by experienced researchers in your field

- rapid publication on acceptance

- support for research data, including large and complex data types

- gold Open Access which fosters wider collaboration and increased citations

- maximum visibility for your research: over $100 \mathrm{M}$ website views per year

At BMC, research is always in progress.

Learn more biomedcentral.com/submissions 